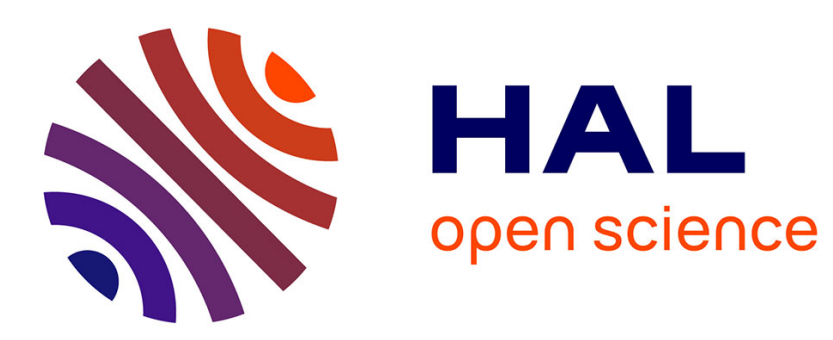

\title{
L'accompagnement de l'innovation par le numérique : quelles opportunités pour les PME ?
}

François Deltour, Sébastien Le Gall, Virginie Lethiais

\section{To cite this version:}

François Deltour, Sébastien Le Gall, Virginie Lethiais. L'accompagnement de l'innovation par le numérique : quelles opportunités pour les PME ?. Reflets et Perspectives de la vie économique, 2020, LVIII (1), pp.101. 10.3917/rpve.591.0101 . hal-02915022v2

\section{HAL Id: hal-02915022 \\ https://hal.science/hal-02915022v2}

Submitted on 23 Feb 2022

HAL is a multi-disciplinary open access archive for the deposit and dissemination of scientific research documents, whether they are published or not. The documents may come from teaching and research institutions in France or abroad, or from public or private research centers.
L'archive ouverte pluridisciplinaire HAL, est destinée au dépôt et à la diffusion de documents scientifiques de niveau recherche, publiés ou non, émanant des établissements d'enseignement et de recherche français ou étrangers, des laboratoires publics ou privés. 


\title{
Version acceptée pour publication
}

François Deltour, Sébastien Le Gall, Virginie Lethiais. L'accompagnement de l'innovation par le numérique : quelles opportunités pour les PME ?. Reflets et Perspectives de la vie économique, De Boeck Supérieur, 2020, LVIII (1), pp.101. 〈10.3917/rpve.591.0101〉.〈hal-02915022〉

\section{L'accompagnement de l'innovation par le numérique : quelles opportunités pour les PME ?}

\author{
François DELTOUR, Sébastien LE GALL, Virginie LETHIAIS ${ }^{1}$
}

\section{Résumé}

L'article vise à mieux comprendre le contexte dans lequel les PME accompagnent leurs innovations par le numérique. La recherche interroge en particulier le rôle du niveau d'appropriation des outils numériques au sein de l'entreprise et de la localisation de la PME sur cet accompagnement. L'analyse empirique s'appuie sur les données collectées en 2015 auprès de 711 PME innovantes et localisées dans des espaces de densité variable au sein de la région Bretagne (France). Il ressort que l'accompagnement des innovations par le numérique est loin d'être systématique. La modélisation économétrique montre que le profil numérique général des PME affecte positivement la probabilité d'accompagner ses innovations par le numérique. Ceci traduit un effet accélérateur pour les PME numérisées, dont ne bénéficient pas celles qui le sont peu. Les résultats montrent aussi un effet de décrochage pour les PME localisées dans les espaces de faible densité, qui investissent moins dans le numérique pour accompagner leurs innovations que celles localisées dans les grands pôles urbains.

\section{Mots clefs}

Innovation, Numérique, Localisation, PME

\footnotetext{
${ }^{1}$ François Deltour est maître de conférences à IMT Atlantique, chercheur en management des systèmes d'information au laboratoire LEMNA et membre associé au GIS Marsouin. francois.deltour@imt-atlantique.fr

Sébastien Le Gall est maître de conférences HDR à l'Université Bretagne Sud, chercheur en management stratégique au laboratoire LEGO et membre du GIS Marsouin. sebastien.le-gall@ univ-ubs.fr

Virginie Lethiais est maître de conférences HDR à IMT Atlantique, chercheur en économie du numérique au laboratoire LEGO et membre du GIS Marsouin. virginie.lethiais@imt-atlantique.fr
} 


\begin{abstract}
The article aims to better understand how SMEs are supporting their innovation performance through digital technology. We wonder if this digital support depends on the firm's general digital profile and on the firm's location. The empirical analysis relies on data collected in 2015 from 711 innovative SMEs located in areas of varying density within the Brittany region (France). It shows that digital support for innovation is far from being systematic. Econometric models show that SMEs' level of IT appropriation positively affects the probability of supporting their innovations through digital technology. This result indicates an accelerating effect for highly digitalised SMEs, from which low digitalised ones do not benefit. The models also show a dropout effect for SMEs located in low-density areas, which invest less in digital technology to support their innovations than those located in large urban centres.
\end{abstract}

\title{
Key Words
}

Innovation, Digital technologies, Location, Small businesses

Codes JEL : D21, O31, R30 


\section{Introduction}

Acteurs clés de l'économie, les Petites et Moyennes Entreprises (PME) doivent faire face à de multiples défis induits par la mondialisation et la transformation numérique des économies (OCDE, 2017). L'innovation constitue pour les PME une réponse à ces défis, même si ces dernières parviennent moins à innover que les entreprises de grande taille (Eurostat, 2014).

Les technologies numériques sont reconnues comme des leviers d'innovation, en particulier dans les PME (Deltour et Lethiais, 2014). La question de la capacité des PME à mobiliser les outils numériques pour accompagner leurs innovations mérite donc d'être creusée. Dans la plupart des travaux sur les effets des technologies numériques sur l'innovation, c'est la superposition d'une stratégie d'innovation et de pratiques numériques qui est mesurée (Higón, 2012 ; Santoleri, 2015). Rien n'indique en effet clairement que les technologies utilisées au sein de l'entreprise sont effectivement mobilisées pour soutenir les innovations mises en œuvre.

Un des principaux apports de notre recherche repose sur la richesse et l'originalité des données collectées et exploitées : nous mesurons non seulement les pratiques numériques des PME mais aussi la mobilisation du numérique par l'entreprise comme un outil d'accompagnement de sa stratégie d'innovation. Notre objectif est de mieux comprendre le contexte dans lequel les PME accompagnent leurs innovations par le numérique. Nous déclinons cet objectif en une question de recherche générale : quels sont les principaux déterminants de l'accompagnement par le numérique de l'innovation des PME?

Parmi les différents déterminants, nous interrogeons l'influence du profil numérique de la PME. Observe-t-on un effet de rattrapage des PME peu numérisées, qui traduirait un usage accru et/ou des investissements dans le numériques dédiés à l'innovation pour ces PME ? Ou à l'inverse, observe-t-on un effet accélérateur, à savoir un accompagnement de l'innovation par le numérique d'autant plus intense que la PME est fortement numérisée ?

Nous portons également une attention particulière au rôle de la localisation de la PME sur l'accompagnement par le numérique de l'innovation. Les technologies numériques sont en effet perçues comme des outils permettant de s'affranchir des contraintes de la distance (Deltour et al., 
2016 ; Torre, 2014). Dans ce cas, les PME innovantes localisées en milieu rural ont-elles tendance à compenser leur isolement géographique par un usage accru et des investissements spécifiques dans le numérique (effet compensateur) ? Au contraire, observe-t-on plutôt un effet de décrochage de ces PME isolées géographiquement, à savoir un accompagnement moindre par les outils numériques?

Dans une première partie, nous présentons les enjeux d'un accompagnement de l'innovation par le numérique. Nous détaillons dans une deuxième partie la méthodologie, à savoir la collecte des données, le modèle mobilisé et les variables retenues pour évaluer les principaux déterminants de l'accompagnement de l'innovation par le numérique. Nous présentons enfin les résultats et les discutons dans une troisième partie.

\section{Les enjeux de l'accompagnement de l'innovation par le numérique}

Les technologies numériques modifient la manière dont l'entreprise conduit l'innovation (Yoo et al., 2012 ; Barlatier, 2016). Elles sont, d'une part, des éléments constitutifs des innovations dont les fonctionnalités se retrouvent enrichies. Elles sont, d'autre part, mobilisées pour activer et combiner des ressources internes ou externes nécessaires au processus d'innovation.

Chatterjee et al. (2020) rappellent les potentialités fondamentales permises par les technologies de l'information dans les organisations, en termes de gestion des connaissances, des collaborations et des processus. Ils soulignent que ces potentialités peuvent participer à réaliser les objectifs que se fixent les organisations, notamment l'innovation. Les auteurs montrent que l'alignement de ces potentialités (via leur renforcement mutuel et leur combinaison créatrice de synergies) combiné à la nécessaire prise de risque de toute nouveauté, soutient l'innovation dans les organisations.

La mise en œuvre des potentialités des technologies numériques se réalise aussi bien en interne, au sein de l'entreprise, que de manière ouverte sur son environnement et ses partenaires extérieurs. Ainsi, les technologies de l'information rendent les entreprises plus « extraverties» (Tambe et al., 2012), c'est-à-dire capables d'être plus en prise avec leur environnement dans leur processus d'innovation. 
Les coopérations externes se trouvent ainsi facilitées par les outils de communication, voire par certaines technologies numériques dédiées à l'innovation (Ben Mahmoud-Jouini, 2016) : outils permettant l'identification de sources externes (outils de recherche, plateformes d'intermédiation, réseaux sociaux...) et outils d'exploration partenariale (plateformes partagées de conception, de simulation et de prototypage, bases de données...).

Ces travaux mettent en avant l'enjeu que constitue le numérique comme moteur et ou comme facilitateur des stratégies d'innovation des entreprises. Dans la continuité de ces travaux, nous considérons les technologies numériques comme outil d'accompagnement de l'innovation et questionnons les déterminants de cet accompagnement.

Un regard particulier est porté sur le profil numérique général de la firme, qui affecte nécessairement la capacité à mobiliser les outils numériques ou à investir dans de nouveaux outils pour accompagner ses innovations (Deltour et al., 2016). Une mobilisation plus importante du numérique par les PME les moins numérisées laisserait supposer qu'un rattrapage est possible de la part de ces entreprises. A l'inverse, une plus forte mobilisation des PME les plus numérisées traduirait l'existence d'un effet accélérateur, préjudiciable aux entreprises ayant déjà pris du retard dans leur transformation numérique.

Parmi les divers enjeux liés à l'accompagnement de l'innovation par le numérique, la littérature met en évidence la capacité qu'offrent les technologies du numérique de s'affranchir des contraintes de la distance (Barlatier, 2016 ; Torre, 2014) et de mieux tirer parti de son environnement (Tambe et al., 2012). Nous portons ainsi une attention particulière à l'influence de la localisation de la PME sur la probabilité d'un accompagnement de l'innovation par le numérique.

\section{La méthode de recherche}

Au regard des enjeux de l'accompagnement de l'innovation par le numérique pour les PME, nous déployons une investigation quantitative permettant d'en identifier les principaux déterminants. Après avoir détaillé la collecte des données, nous présentons la modélisation économétrique suivie, puis les variables explicatives retenues. 


\subsection{LA COLLECTE DES DONNEES}

L'investigation quantitative s'appuie sur les données d'une enquête réalisée par le GIS Marsouin $^{2}$ en 2015 auprès des entreprises de 10 à 250 salariés appartenant aux secteurs de l'industrie, du commerce et des services (à l'exception de l'agriculture et des services publics), localisées en région Bretagne.

L'enquête repose sur un questionnaire soumis à toutes les PME recensées dans les registres de la Chambre de Commerce et de l'Industrie (CCI) de la région, soit 7251 entreprises. 1469 questionnaires complets ont été collectés, ce qui représente un taux de réponse de près de $20 \%$. Le répondant est, dans la majorité des cas, le dirigeant de l'entreprise ou le responsable administratif ou/et financier. La méthode des quotas a été utilisée afin d'obtenir un échantillon représentatif des PME bretonnes selon le secteur d'activité, la CCI de rattachement et l'effectif salarial.

L'enquête, qui interroge d'abord chaque PME sur son activité et sa situation générale, nous indique si la PME a innové au cours des deux dernières années. L'innovation est appréhendée dans l'enquête de manière large : une PME est considérée innovante lorsqu'elle déclare avoir mis sur le marché un nouveau produit ou mis en œuvre de nouveaux procédés de production, de nouvelles méthodes de distribution ou une nouvelle activité de soutien pour les produits/services.

Il est ensuite demandé aux PME innovantes, d'une part, si elles ont coopéré dans le cadre de ces innovations et, d'autre part, si elles ont accompagné leurs innovations par du numérique. Cet accompagnement recouvre deux pratiques différentes, mais possiblement complémentaires :

- un investissement spécifique dans le numérique ;

- un usage plus développé des outils numériques déjà présents dans l’entreprise.

Nous cherchons alors à expliquer les pratiques d'accompagnement déclarées par les PME innovantes de notre échantillon, soit 711 entreprises sur les 1469 répondantes à l'enquête.

\subsection{LA MODELISATION UTILISEE}

Nous construisons une modélisation économétrique qui estime, sur cet échantillon de 711 PME innovantes, la probabilité d'accompagner ses innovations par le numérique, en

\footnotetext{
${ }^{2}$ www.marsouin.org
} 
distinguant l'accompagnement qui consiste uniquement en un usage plus développé des outils existants, de l'accompagnement qui inclut un investissement dans de nouveaux outils. Nous utilisons pour cela un modèle Logit multinomial dont la variable expliquée peut prendre trois modalités :

1- accompagner l'innovation par un usage plus développé des outils numériques existants ;

2- accompagner l'innovation par un investissement dans le numérique ;

3- ne pas accompagner l'innovation (variable de référence).

\subsection{LES VARIABLES EXPLICATIVES RETENUES}

La variable expliquée est estimée en fonction du niveau général d'appropriation du numérique dans la PME et de sa localisation, ainsi que de plusieurs variables de contrôle (la nature des innovations, la probabilité de coopérer pour ses innovations, les effectifs, le secteur d'activité).

\subsubsection{L’appropriation du numérique}

Les ressources numériques des firmes sont caractérisées selon trois dimensions complémentaires, qui reflètent à la fois les équipements, les compétences informatiques et pratiques de l'entreprise, selon l'approche de Aral et Weill (2007).

Les actifs TIC sont appréhendés par la diversité des usages numériques, qui peut prendre quatre modalités en fonction du nombre d'outils numériques différents utilisés dans l'entreprise parmi une liste de onze ${ }^{3}$.

Les compétences informatiques internes à l'entreprise sont mesurées par une variable qui peut prendre trois modalités suivant qu'il existe un service informatique constitué dans l'entreprise, qu'au moins un salarié de l'entreprise est diplômé du supérieur en informatique ou qu'aucune de ces compétences n'existe en interne.

Enfin, nous intégrons dans les modèles un troisième facteur d'appropriation d'Internet qui reflète les pratiques au sein de l'entreprise. Cette appropriation d'Internet est mesurée par la part

${ }^{3}$ Les 11 outils TIC ou usages des TIC considérés dans l'enquête sont les suivants : EDI (Échange de Données Informatisées), intranet, listes de diffusion, agendas partagés, espaces de travail partagés, logiciels de définition et de gestion des processus, logiciels métiers, certificats électroniques, site Web, réseaux sociaux, visioconférence. 
des salariés qui utilisent Internet tous les jours ou presque, codée en trois modalités : tous, plus de $25 \%$, moins de $25 \%$. Ces trois variables, très dépendantes les unes des autres sont intégrées alternativement dans la modélisation.

\subsubsection{La localisation de la PME}

L'INSEE décompose l'espace en 9 catégories, qui permettent d'appréhender l'existence de pôles urbains, mais aussi l'influence de ces pôles sur les communes périphériques. Nous fusionnons certaines de ces catégories afin d'adopter une décomposition de l'espace en 3 modalités :

- les grands pôles urbains, constitués des communes qui regroupent plus de 10000 emplois ;

- le périurbain, qui regroupe l'ensemble des communes sous influence des grands pôles urbains, par le fait que plus de $40 \%$ de leur population travaille dans ces grands pôles ;

- l'espace rural, qui rassemble les 6 autres catégories, soit l'ensemble des communes situées en dehors des grandes aires urbaines, et qui ne subissent pas l'influence de ces grandes aires.

Cette décomposition s'appuie sur la concentration des emplois, et constitue une bonne approximation du degré d'urbanisation de l'espace et donc du niveau d'agglomération des ressources que l'entreprise est susceptible de mobiliser et des externalités locales dont elle peut bénéficier. La figure 1 représente la partition de la région Bretagne selon ce découpage en trois modalités. 

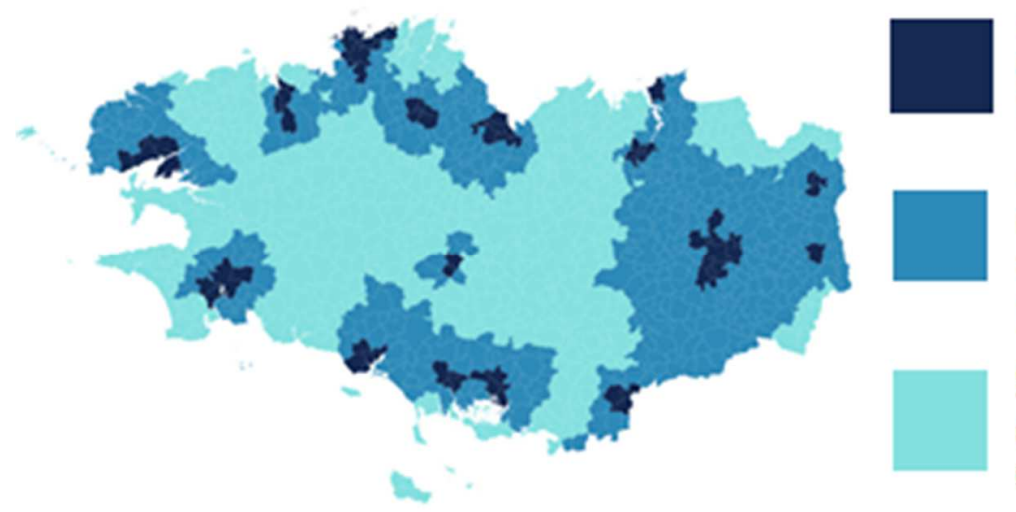

Grandes aires urbaines: communes d'au moins 10000 emplois

Aires périurbaines : regroupant les communes sous influence des grandes aires urbaines, où plus de $40 \%$ de la population va travailler dans l'aire urbaine

Zone rurale : regroupant les communes sitvees hors des grandes aires urbaines et non sujettes à leur influence

\subsubsection{Les variables de contrôle}

Quatre variables de contrôle sont également intégrées dans les modèles, afin de caractériser l'entreprise, mais aussi la nature de ses innovations.

La nature des innovations est appréhendée d'une part par une variable en trois modalités qui indique si l'entreprise innove uniquement en procédé, uniquement en produit, ou si elle déclare les deux types d'innovation et d'autre part par une variable binomiale qui prend la valeur 1 si la PME déclare avoir coopéré pour ses innovations et 0 sinon.

Enfin, nous intégrons deux variables qui caractérisent la PME : sa taille, mesurée par l'effectif salarié, en 3 modalités : entre 10 et 19 salariés, entre 20 et 49 salariés, 50 salariés et plus ; le secteur d'activité, en 6 modalités (industrie, transport, commerce et hôtellerie-restauration, construction, activités spécialisées scientifique et technique information communication, activités immobilières financières et d'assurance et autres services). 


\section{Résultats et discussion}

\subsection{UN ACCOMPAGNEMENT NON SYSTEMATIQUE DE L'INNOVATION PAR LE NUMERIQUE}

Le premier résultat qui ressort de l'enquête est que l'accompagnement des innovations par le numérique est loin d'être systématique. Seule une PME sur deux (52\%) accompagne l'innovation par le numérique. Cet accompagnement passe par un investissement spécifique pour $43 \%$ des entreprises interrogées, par un usage plus développé des technologies déjà présentes dans l'entreprise pour $44 \%$; plus d'un tiers des firmes (35,3\% des firmes interrogées) associent l'investissement et l'usage pour accompagner leurs innovations. À cet égard, on observe que l'investissement dans le numérique et l'usage plus développé des outils existants sont très complémentaires : si une part importante des PME combine en effet les deux (35\%), une autre part importante (45\%) n'accompagne pas du tout leurs innovations par le numérique (Graphique 1).

Graphique 1 : L'accompagnement de l'innovation par le numérique

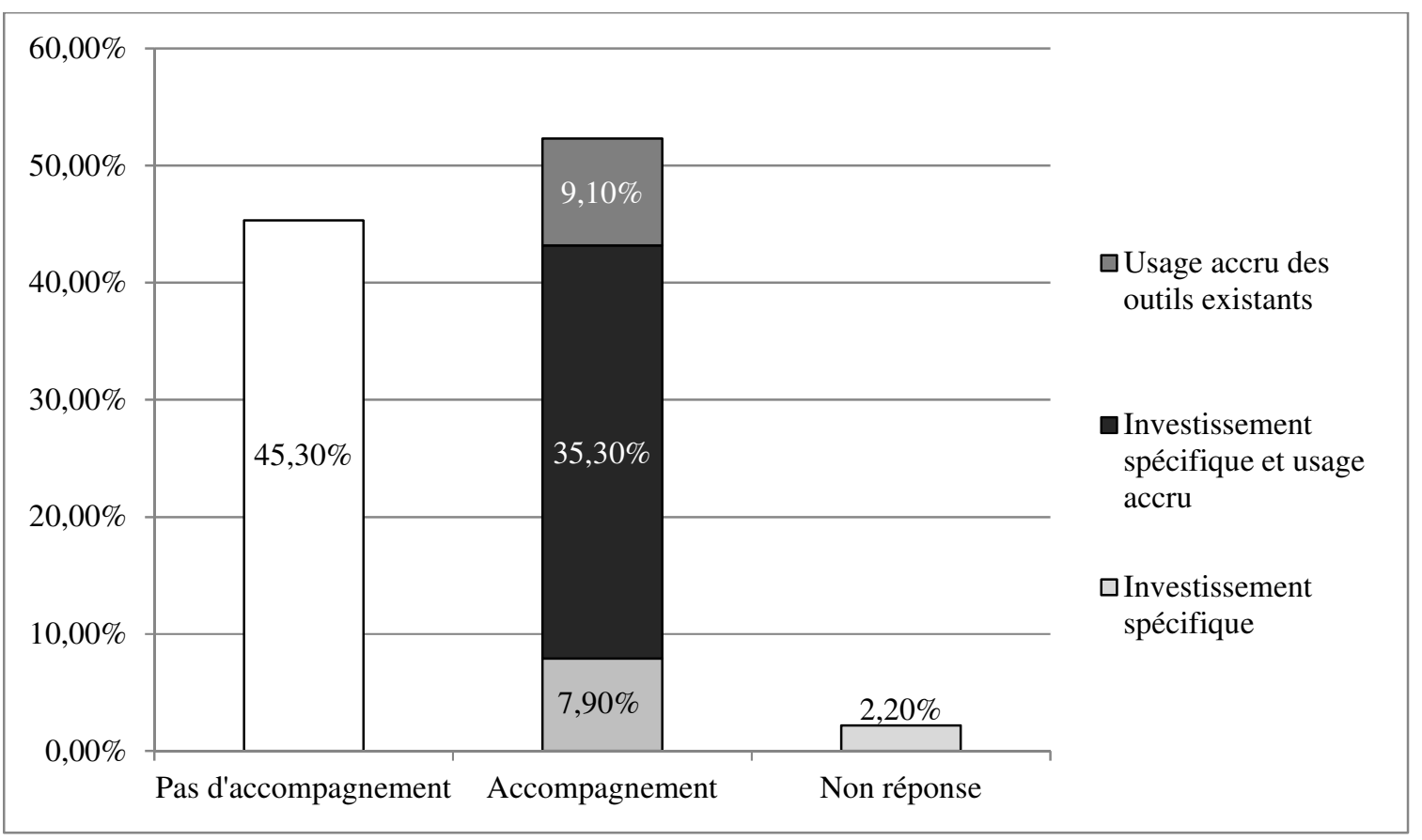

Ce constat va dans le sens d'autres déjà établis qui mettent en évidence le retard des PME en matière d'adoption des technologies numériques (Kocoglu et Moatty, 2010). Selon le rapport de l'OCDE (2017), si le retard est minime pour ce qui est de la simple connectivité et de la présence sur Internet, l'écart se creuse par rapport aux grandes entreprises pour des applications plus sophistiquées. Il est notamment souligné que «le manque d'investissement dans les processus 
d'innovation et les capacités organisationnelles internes empêche les PME de mettre pleinement à profit les nouvelles technologies du numérique pour renforcer leur analyse des données et accroître leur participation aux réseaux de connaissance » (2017, p. 16). L'essor des technologies numérique peut alors être source de perturbations pour les PME en accélérant «la contestabilité des marchés, l'obsolescence des connaissances, des compétences et des modèles économiques » (ibid.).

Afin de mieux apprécier ce retard des PME, nos modèles mettent en évidence les déterminants de l'accompagnement de l'innovation par le numérique.

\subsection{L'APPROPRIATION DU NUMERIQUE : UN EFFET ACCELERATEUR}

Nous testons l'effet du profil numérique de la PME via trois mesures différentes : la diversité des usages des outils numériques (modèle 1), l'appropriation d'Internet par les salariés (modèle 2) et les compétences internes en informatique (modèle 3).

Les résultats des modèles 1 à 3 sont présentés dans le tableau 1. Pour chaque modèle, la première colonne indique l'impact des variables considérées sur la probabilité d'accompagner ses innovations par un usage plus développé des outils numériques existants (par rapport à ne pas accompagner ses innovations par le numérique) ; la seconde colonne indique l'impact des variables considérées sur la probabilité d'accompagner ses innovations par un investissement dans le numérique (par rapport à ne pas accompagner ses innovations par le numérique) 


\begin{tabular}{|c|c|c|c|c|c|c|}
\hline \multirow{2}{*}{$\begin{array}{l}\text { Tableau } 1 \text { : Les } \\
\text { modèles } \\
\text { d'estimation de la } \\
\text { probabilité } \\
\text { d'accompagner } \\
\text { par le } \\
\text { numériquevariables }\end{array}$} & \multicolumn{2}{|c|}{ Modèle 1} & \multicolumn{2}{|c|}{ Modèle 2} & \multicolumn{2}{|c|}{ Modèle 3} \\
\hline & $\begin{array}{l}\text { Accompagnement } \\
\text { par l'usage } \\
\text { uniquement }\end{array}$ & $\begin{array}{l}\text { Accompagnement } \\
\text { par l'investissement }\end{array}$ & $\begin{array}{l}\text { Accompagnement } \\
\text { par l'usage } \\
\text { uniquement }\end{array}$ & $\begin{array}{l}\text { Accompagnement } \\
\text { par l'investissement }\end{array}$ & $\begin{array}{l}\text { Accompagnement } \\
\text { par l'usage } \\
\text { uniquement }\end{array}$ & $\begin{array}{l}\text { Accompagnement } \\
\text { par l'investissement }\end{array}$ \\
\hline \multicolumn{7}{|l|}{ Localisation de la firme } \\
\hline Grands pôles urbains & Ref. & Ref. & Ref. & Ref. & Ref. & Ref. \\
\hline Péri-urbain & NS & $0.58 * * *$ & NS & $0.62 * *$ & NS & $0.58^{* * *}$ \\
\hline Rural & NS & $0.60 *$ & NS & $0.61 * *$ & NS & $0.58^{* * *}$ \\
\hline \multicolumn{7}{|l|}{ Profil numérique de la firme } \\
\hline \multicolumn{7}{|c|}{ Diversité des usages : nombre d'outils utilisés parmi 11} \\
\hline Très diversifiés & $2.56 *$ & $2.85 * * *$ & & & & \\
\hline Diversifiés & $1.60 * * *$ & $3.54 * *$ & & & & \\
\hline Peu diversifiés & NS & NS & & & & \\
\hline Très peu diversifiés & Ref. & Ref. & & & & \\
\hline \multicolumn{7}{|c|}{ Appropriation d'Internet : part des salariés qui utilisent Internet tous les jours ou presque } \\
\hline Tous & & & $2.98 * *$ & $2.88^{* * *}$ & & \\
\hline Plus de 25\% & & & NS & NS & & \\
\hline Moins de $25 \%$ & & & Ref. & Ref. & & \\
\hline \multicolumn{7}{|l|}{ Compétences internes } \\
\hline Service informatique & & & & & NS & $1.90 * * *$ \\
\hline Salarié diplômé & & & & & NS & NS \\
\hline Aucune compétence & & & & & Ref. & Ref. \\
\hline \multicolumn{7}{|c|}{ Caractéristiques de l'innovation } \\
\hline \multicolumn{7}{|c|}{ Type d'innovation } \\
\hline Les deux & $1.97 *$ & $2.10^{* * *}$ & $2.04 * * *$ & $2.24 * *$ & $4.75 *$ & $2.18 * * *$ \\
\hline Procédé & $4.54^{* * *}$ & $2.89 * * *$ & $5.06 * * *$ & $3.32 * * *$ & $2.18^{* * *}$ & $3.01 * * *$ \\
\hline Produit & Ref. & Ref. & Ref. & Ref. & Ref. & Ref. \\
\hline \multicolumn{7}{|c|}{ Coopération pour l'innovation } \\
\hline Oui & NS & $1.65^{* * *}$ & NS & $1.70 * * *$ & NS & $1.75 * * *$ \\
\hline \multicolumn{7}{|l|}{ Caractéristiques de la firme } \\
\hline \multicolumn{7}{|l|}{ Effectif salarié } \\
\hline 50-250 salariés & NS & $1.71 * *$ & $2.52 * * *$ & $2.02 *$ & NS & $1.98 * * *$ \\
\hline 20-50 salariés & NS & NS & NS & NS & NS & NS \\
\hline
\end{tabular}




\begin{tabular}{|c|c|c|c|c|c|c|}
\hline 10-19 salariés & Ref. & Ref. & Ref. & Ref. & Ref. & Ref. \\
\hline \multicolumn{7}{|l|}{ Secteurs d'activités } \\
\hline $\begin{array}{c}\text { Commerce hôtellerie- } \\
\text { restauration }\end{array}$ & $0.51 *$ & $2.38 * * *$ & $0.48 *$ & $2.10 * * *$ & NS & $2.34 * * *$ \\
\hline Transport & NS & NS & NS & NS & NS & NS \\
\hline Construction & NS & NS & NS & NS & NS & NS \\
\hline ASSTIC $^{a}$ & NS & $2.49 * * *$ & NS & NS & NS & $2.64 * * *$ \\
\hline Autres services & NS & $1.90 *$ & NS & NS & $1.94 *$ & $2.21 * *$ \\
\hline Industrie & Ref. & Ref. & Ref. & Ref. & Ref. & Ref. \\
\hline
\end{tabular}

${ }^{a}$ Activités spécialisées scientifiques et techniques, information, communication.

Les étoiles indiquent le niveau de significativité de la variable ou modalité : *** pour $1 \%$, ** pour $5 \%$ et * pour $10 \%$.

NS indique que la variable ou modalité n'est pas significative. 


\begin{tabular}{|c|c|c|c|c|c|c|}
\hline \multirow{2}{*}{ Variables } & \multicolumn{2}{|c|}{ Modèle 1} & \multicolumn{2}{|c|}{ Modèle 2} & \multicolumn{2}{|c|}{ Modèle 3} \\
\hline & $\begin{array}{l}\text { Accompagnement } \\
\text { par l'usage } \\
\text { uniquement }\end{array}$ & $\begin{array}{l}\text { Accompagnement } \\
\text { par } \\
1 \text { 'investissement }\end{array}$ & $\begin{array}{l}\text { Accompagnement } \\
\text { par 1'usage } \\
\text { uniquement }\end{array}$ & $\begin{array}{l}\text { Accompagnement } \\
\text { par } \\
\text { l'investissement }\end{array}$ & $\begin{array}{l}\text { Accompagnement } \\
\text { par l'usage } \\
\text { uniquement }\end{array}$ & $\begin{array}{l}\text { Accompagnement } \\
\text { par } \\
\text { l'investissement }\end{array}$ \\
\hline \multicolumn{7}{|c|}{ Localisation de la firme } \\
\hline Grands póles urbains & Ref. & Ref. & Ref. & Ref. & Ref. & Ref. \\
\hline Péri-urbain & NS & $0.58 * * *$ & NS & $0.62 * *$ & NS & $0.58^{* * *}$ \\
\hline Rural & NS & $0.60^{*}$ & NS & $0.61 * *$ & NS & $0.58 * * *$ \\
\hline \multicolumn{7}{|c|}{ Profil numérique de la firme } \\
\hline \multicolumn{7}{|c|}{ Diversité des usages : nombre d'outils utilisés parmi 11} \\
\hline Très diversifiés & $2.56^{*}$ & $2.85 * * *$ & & & & \\
\hline Diversifiés & $1.60^{* * *}$ & $3.54 * *$ & & & & \\
\hline Peu diversifiés & NS & NS & & & & \\
\hline Très peu diversifiés & Ref. & Ref. & & & & \\
\hline \multicolumn{7}{|c|}{ Appropriation d'Internet : part des salariés qui utilisent Internet tous les jours ou presque } \\
\hline Tous & & & $2.98 * *$ & $2.88^{* * *}$ & & \\
\hline Plus de $25 \%$ & & & NS & NS & & \\
\hline Moins de 25\% & & & Ref. & Ref. & & \\
\hline \multicolumn{7}{|l|}{ Compétences internes } \\
\hline Service informatique & & & & & NS & $1.90 * * 8$ \\
\hline Salarié dédié & & & & & NS & NS \\
\hline Aucune compétence & & & & & Ref. & Ref. \\
\hline \multicolumn{7}{|c|}{ Caractéristiques de l'innovation } \\
\hline \multicolumn{7}{|l|}{ Type d'innovation } \\
\hline Les deux & $1.97 *$ & $2.10^{* * *}$ & $2.04^{* * * 8}$ & $2.24 * *$ & $4.75 *$ & $2.18^{* * *}$ \\
\hline Procédé & $4.54 * * *$ & $2.89 * * *$ & $5.06^{* * 8}$ & $3.32 * * *$ & $2.18^{* * *}$ & $3.01 * * *$ \\
\hline Produits & Ref. & Ref. & Ref. & Ref. & Ref. & Ref. \\
\hline \multicolumn{7}{|c|}{ Coopération pour l'innovation } \\
\hline $\mathrm{Oui}$ & NS & $1.65^{* * *}$ & NS & $1.70^{* * *}$ & NS & $1.75^{* * *}$ \\
\hline \multicolumn{7}{|c|}{ Caractéristiques de la firme } \\
\hline \multicolumn{7}{|l|}{ Effectif salarié } \\
\hline $50-250$ salariés & NS & $1.71 * *$ & $2.52 * * *$ & $2.02 *$ & NS & $1.98 * * *$ \\
\hline $20-50$ salariés & NS & NS & NS & NS & NS & NS \\
\hline 10-19 salariés & Ref. & Ref. & Ref. & Ref. & Ref. & Ref. \\
\hline \multicolumn{7}{|l|}{ Secteurs d'activités } \\
\hline $\begin{array}{r}\text { Commerce hôtellerie- } \\
\text { restauration } \\
\end{array}$ & $0.51^{*}$ & $2.38 * * 8$ & $0.48 *$ & $2.10^{* * *}$ & NS & $2.34^{* * *}$ \\
\hline Transport & NS & NS & NS & NS & NS & NS \\
\hline Construction & NS & NS & NS & NS & NS & NS \\
\hline$A S S T I C^{a}$ & NS & $2.49 * * *$ & NS & NS & NS & $2.64^{* * *}$ \\
\hline Autres services & NS & $1.90^{*}$ & $\mathrm{NS}$ & NS & $1.94 *$ & $2.21 * *$ \\
\hline Industrie & Ref. & Ref. & Ref. & Ref. & Ref. & Ref. \\
\hline
\end{tabular}

a- Activités spécialisées scientifiques et techniques, information, communication 
Afin de faciliter la lecture, nous avons fait le choix de ne pas présenter dans les tableaux de résultats les valeurs des coefficients estimés, dont l'interprétation n'est pas directe dans les modèles Logit. Nous présentons, le niveau de significativité de chaque variable ou la modalité de la variable (une étoile pour $10 \%$, deux pour $5 \%$ et trois pour $1 \%$ ), et les rapports de cotes ${ }^{4}$ pour les variables ou modalités significatives. Pour les variables multinomiales, la modalité de référence est notée «Réf ». Une variable ou modalité non significative est indiquée par un 'NS'. Une case grisée indique que la variable ou modalité n'a pas été intégrée dans le modèle.

Dans le tableau 1, il apparaît que le profil numérique de la PME affecte la probabilité d'accompagner ses innovations par le numérique. Quel que soit le type d'accompagnement (usage ou investissement), la probabilité d'accompagner par le numérique est multipliée par plus de 1,5 si la PME a des usages diversifiés ou très diversifiés des outils numériques, par rapport à une entreprise qui utiliserait très peu d'outils numériques (modèle 1). L'appropriation d'Internet est également significative (modèle 2) : le fait que tous les salariés utilisent Internet tous les jours ou presque multiplie par près de 3 la probabilité d'accompagner par le numérique (usage et investissement) par rapport à une PME caractérisée par un niveau d'appropriation faible (moins de $25 \%$ des salariés).

Ces résultats révèlent un effet accélérateur entre l'appropriation générale du numérique et sa mobilisation dans le cadre spécifique de l'accompagnement de l'innovation. Un écart se creuse entre les PME «peu numérisées » (qui de ce fait mobilisent peu le numérique pour accompagner leurs innovations) et les PME équipées (qui s'appuient plus naturellement sur ces outils dans leurs processus d'innovation).

On note cependant que la variable de compétence numérique affecte la probabilité d'accompagner par l'investissement, mais pas la probabilité d'accompagner par l'usage (modèle 3). La présence d'un service informatique multiplie par 1,9 la probabilité d'accompagner l'innovation par un investissement spécifique dans le numérique. Si l'absence de compétences internes en informatique ne semble donc pas freiner la mobilisation des outils présents dans

\footnotetext{
${ }^{4}$ Un rapport de cotes égal à x implique que la probabilité que la firme accompagne par le numérique est multipliée par x pour la modalité considérée par rapport à la modalité de référence. Un rapport de cote supérieur à 1 traduit ainsi un effet positif de la variable (ou modalité) sur la probabilité d'accompagner ses innovations par le numérique alors qu'un rapport de cote inférieur à 1 traduit un effet négatif de la variable (ou modalité).
} 
l'entreprise pour accompagner les innovations, la réalisation de nouveaux investissements pour accompagner ses innovations semble, elle, conditionnée à la présence de telles compétences. Ce résultat va dans le sens de celui mis en évidence par Aral et Weill (2007) pour qui la capacité à mobiliser efficacement le numérique passe par la combinaison de compétences internes et d'actifs numériques dans lesquels l'entreprise investit. On observe donc un effet d'accélération de l'accompagnement de l'innovation par un investissement spécifique dans le numérique pour les PME qui disposent d'un service informatique.

\subsection{Localisation des PME : Un EFFET DE DECROCHAGE POUR LES PME hORS DES GRANDS CENTRES URBAINS}

Un résultat qui ressort des modèles 1, 2 et 3 est que la localisation de la PME affecte la probabilité d'investir dans le numérique pour accompagner ses innovations, mais pas la probabilité de les accompagner par un usage plus développé des outils existants.

Les PME localisées dans le péri-urbain et le rural sont moins enclines à investir dans le numérique pour accompagner leurs innovations que les PME localisées dans les grands pôles urbains. Plus précisément, la probabilité d'accompagner l'innovation par un investissement spécifique dans le numérique est près de 2 fois plus faible (rapports de cotes entre 0,58 et 0,62) pour ces PME que pour les PME localisées dans les grands centres urbains. Les travaux de Shearmur (2015) contribuent à expliquer ce résultat. Selon lui, lorsque l'innovation est étroitement liée au marché, et s'appuie sur des connaissances et des informations dont le caractère stratégique suppose une absorption rapide, une localisation dans les métropoles ou les grands centres urbains est un atout. La mobilisation du numérique apparaît donc d'autant plus nécessaire pour accompagner ce type d'innovations. Il apparaît en outre que ce moindre investissement dans le numérique pour accompagner les innovations, de la part des entreprises localisées dans des espaces peu denses, n'est pas compensé par un usage plus important des outils existants. En effet, dans les 3 modèles, la variable de localisation n'a pas d'impact sur la probabilité d'accompagner par l'usage.

Ces effets rendent compte d'un décrochage qui, s'il ne devait pas uniquement se justifier par la nature des innovations développées, pourrait réduire les potentialités qu'offrent les technologies numériques pour les PME qui se situent hors des grands centres urbains. 


\subsection{LES EFFETS DES VARIABLES DE CONTROLE}

La nature des innovations affecte la probabilité d'accompagner ses innovations par le numérique, traduisant un accompagnement plus systématique, d'une part des innovations procédés par rapport aux innovations produits et d'autre part des innovations réalisées en coopération par rapport aux innovations non coopératives. Cela va dans le sens des travaux qui soulignent le rôle du numérique dans l'amélioration de la gestion des processus (Chatterjee et al., 2020), rendant l'accompagnement essentiel dans le cas d'innovations de procédés. De même, l'accompagnement plus systématique des innovations coopératives est justifié par les potentialités offertes par les outils numériques en termes de mobilisation des coopérations externes (Tambe et al., 2012 ; Ben Mahmoud-Jouini, 2016).

Enfin la taille de l'entreprise et son secteur d'activité ne sont pas sans effet, les entreprises de plus grande taille et celles appartenant aux secteurs les plus proches des consommateurs finaux (en particulier Commerce Hôtellerie Restauration) étant plus enclines à accompagner leurs innovations par un investissement dans le numérique.

\section{Conclusion}

Le premier résultat de la recherche est que l'accompagnement de l'innovation par le numérique est loin d'être systématique pour les PME. Nous pouvons y voir la difficulté régulièrement constatée des PME à s'approprier les technologies du numérique, en raison notamment, au-delà des seuls aspects techniques, « des pénuries de compétences et des défaillances au niveau des pratiques de gestion et de la formation des collaborateurs » (OCDE, 2017, p. 18).

Les analyses réalisées à partir des PME innovantes qui déclarent accompagner leur innovation par le numérique rendent également compte d'un écart qui se creuse entre les PME peu numérisées et les PME mieux équipées pour répondre aux besoins d'innovation. La présence d'un service informatique a également un effet très significatif sur le fait d'investir spécifiquement dans le numérique pour innover.

Nous observons enfin un effet de décrochage pour les PME localisées en dehors des grands centres urbains, ces dernières ayant tendance à moins investir dans le numérique pour innover.

Ces résultats sont d'autant plus critiques que conjuguer les technologies du numérique et l'innovation a un effet positif sur la performance (Deltour et Lethiais, 2014). Ils pourraient ainsi 
traduire l'existence d'un cercle vicieux en termes de performance pour les PME peu numérisées et situées en dehors des grands centres urbains.

Apparait ainsi un enjeu de politique publique visant à «l'accompagnement de la transition numérique des PME» (Sénat, 2019). Parmi les différents leviers envisagés, les PME peuvent se saisir de ceux présents dans leur écosystème, soit par l'entraide professionnelle (le réseau des CCI, les fédérations professionnelles, les associations) soit par le recours au marché. Il s'agit de faire évoluer leurs pratiques d'innovation en interne, à la fois sur les procédés, par l'intégration de nouvelles fonctionnalités liées au numérique, et sur les produits, en se saisissant notamment des opportunités qu'ouvrent les plateformes numériques (Sénat, 2019). Pour une performance durable, cette évolution supposera l'internalisation de compétences numériques dédiées à l'innovation et l'existence d'une réelle dynamique d'apprentissage (Aral et Weill, 2007).

Notre recherche n'est pas exempte de limites. La principale porte sur la mesure de l'innovation que nous avons adoptée, qui, si elle nous permet de prendre en compte les comportements d'innovation des PME sur une large échelle, ne nous fournit que peu d'informations sur la nature et l'intensité de l'innovation. Or, comme nous avons pu le noter, une interprétation fine de nos résultats rend nécessaires des investigations supplémentaires. Une question restant en suspens, qui nécessite une approche qualitative, est de mieux comprendre les raisons pour lesquelles les PME localisées en dehors des grands pôles urbains et les PME faiblement numérisées investissent moins dans le numérique pour accompagner leurs innovations.

\section{Références}

ArAl, S., WeILl, P. (2007), "IT Assets, Organizational Capabilities, and Firm Performance: How Resource Allocations and Organizational Differences Explain Performance Variation », Organization Science, 18(5), pp. 763-790.

BARlatier, P. J. (2016), "Management de l'innovation et nouvelle ère numérique - enjeux et perspectives”, Revue Française de Gestion, 254, pp. 55-63.

Ben MAhmoud-Jouini, S. (2016), "Le numérique au service des entités dédiées à l'innovation de rupture”, Revue Française de Gestion, 254 (avril), pp. 65-87.

Chatterjee, S., Moody, G. D., Lowry, P. B., Chakraborty, S., Hardin, A. (2020), "Information Technology and organizational innovation: Harmonious information 
technology affordance and courage-based actualization", Journal of Strategic Information Systems, 29(1), 101596, 1-23.

Deltour, F., LethiAis V., (2014), “L'innovation en PME et son accompagnement par les TIC : quels effets sur la performance ?" Systèmes d'Information et Management, 19(2), pp. 4573.

Deltour, F. Le Gall, S., Lethiais, V. (2016), « Le numérique modifie-t-il le lien entre territoire et innovation? Une étude empirique sur les PME », Revue d'Économie Industrielle, 156, 17-49.

EUROSTAT. (2014). Key figures on Europe - 2014 edition, Report.

HIGÓN, D. (2012), "The impact of ICT on innovation activities: Evidence for UK SMEs", International Small Business Journal, 30(6), pp. 684-699.

Kocoglu Y., MoAtTy F. (2010), “Diffusion et combinaison des TIC au sein des entreprises en 2006 : les réseaux, la gestion des données et l'intégration par les ERP”, Réseaux, $\mathrm{n}^{\circ} 162$, pp. 38-71.

OCDE (2017), Renforcer les contributions des PME dans une économie mondialisée et numérique, rapport.

SANTOLERI, P. (2015), "Diversity and Intensity of Information and Communication Technologies Use and Product Innovation: Evidence from Chilean Micro-Data". Economics of Innovation and New Technology, 24(6), pp. 550-568.

SENAT (2019), L'accompagnement de la transition numérique des PME : comment la France peutelle rattraper son retard? rapport, 4 juillet

SHEARMUR, R (2015), "Far from the madding crowd: slow innovators, information value and the geography of innovation", Growth and Change, 46(3), pp. 424-442.

TAMBE, P., HitT, L., BRYNJOlfSSON, E. (2012), “The extroverted firm: How external information practices affect innovation and productivity”, Management Science, 58(5), pp. 843-859.

TORRE, A. (2014), «Relations de proximité et comportements d'innovation des entreprises des clusters », Revue Française de Gestion, 242, pp. 49-80. 
Yoo, Y., Boland, JR R.J., Lyytinen, K., MajchrZaK, A, (2012), “Organizing for innovation in the digitized world", Organization Science, 23(5), pp. 1398-1408. 